\title{
RECOMBINANT EXPRESSION AND FUNCTIONAL CHARACTERIZATION OF ANTIMICROBIAL PEPTIDE CRUSTIN FROM ARTEMIA SALINA
}

\author{
Jayesh $\mathbf{P}^{1^{*}}$, Sruthy K S ${ }^{1^{*}}$, I S Bright Singh ${ }^{2^{*}}$ and Rosamma Philip ${ }^{1^{* \S}}$ \\ ${ }^{1}$ Department of Marine Biology, Microbiology \& Biochemistry, School of Marine Sciences, \\ Cochin University of Science and Technology, Fine Arts Avenue, Kochi 16, Kerala, India \\ ${ }^{2}$ National Centre for Aquatic Animal Health, Cochin University of Science and Technology, \\ Fine Arts Avenue, Kochi 16, Kerala, India
}

\begin{abstract}
Crustins are cationic Antimicrobial peptides (AMPs) containing a whey acidic protein (WAP) domain at the C-terminus. The present study is focused at recombinant production and functional characterization of crustin-I AMP isoform from the brine shrimp, Artemia salina (As-Crustin). AsCrustin sequence was highly similar to the crustins of Scylla serrata and Scylla paramamosain and shared $88 \%$ identity among each other. The mature peptide of As-Crustin is composed of 90 amino acids with a molecular weight of $10 \mathrm{kDa}, \mathrm{p} I$ of 8.0 and net charge +3 . The WAP domain was the only domain found in the As-Crustin sequence obtained in the present study. This domain forms a tightly packed structure described on PROSITE as a four-disulphide core (4DSC). Peptide models of Ascrustin composed of a tightly coiled structure enclosing two $\beta$-sheets but no helices or possibly a loop protein. Active peptide of As-Crustin with WAP domain was produced by heterologous expression in E. coli, Rosettagami B DE3 pLysS using the pET-32a+ vector. The purified and refolded rAs-Crustin protein exhibited antimicrobial activity at a concentration of $2.5 \mu \mathrm{M}$ against Gram-negative bacteria Pseudomonas aeruginosa and Edwardsiella tarda and Gram-positive bacteria Bacillus cereus and Staphylococcus aureus. Recombinant peptide was not hemolytic or cytotoxic even at a concentration of $6 \mu \mathrm{M}$. Results from the functional studies propose that rAs-Crustin is a promising potent therapeutic agent against bacterial infection.
\end{abstract}

Keywords: Antimicrobial peptides; Crustin; Artemia salina; Innate Immunity; Recombinant protein.

*These authors have contributed equally to this work.

${ }^{\S}$ Corresponding author. Tel: + 914842368120, Fax: + 914842381120

E-mail address: rosammap@gmail.com 